HNO 2021 · 69:416-421

https://doi.org/10.1007/s00106-021-01013-4

Angenommen: 26. Januar 2021

Online publiziert: 17. März 2021

(c) Springer Medizin Verlag GmbH, ein Teil von Springer Nature 2021

Überall auf der Erde durchströmen Flüsse die Länder und bringen Geröll aus den Bergen in die Meere. Dabei nehmen sie Wasser und Geröll kleinerer Flüsse aus anderen Ländern auf und formen zusammen neues Land in ihren Mündungsdeltas. Ein solcher Strom mit seinen vielen Nebenflüssen ist wie das Bild der heutigen DGHNO-KHC, die seit 1976 ihre Geschäftsstelle in Bonn am Rhein hat. Gegründet wurde ihr erster Vorläufer, die Gesellschaft Deutscher Hals-, Nasen- und Ohrenärzte, 1921 in Nürnberg. Nach dem Krieg fand 1949 in Karlsruhe die Neugründung der Gesellschaft Deutscher Hals-Nasen-Ohrenärzte statt. Im Jahr 1968 wurde diese in Deutsche Gesellschaft für Hals-Nasen-Ohren-Heilkunde, Kopf- und HalsChirurgie umbenannt.

Dieser Bericht versucht vom geografischen Aspekt her etwas über die Herkunft der "Nebenflüsse“ zu erfahren, die den heutigen "Strom“ DGHNOKHC mit Quellen aus vielen Ländern der Welt vernetzen und bereichern. Auf die Aktivitäten der zahlreichen Gesellschaften während ihrer Kontakte wird nicht eingegangen. Die wesentliche Quelle von Unterlagen für diese Recherche war die Geschäftsstelle der DGHNO-KHC mit ihrem Archiv in Bonn. Hier findet sich seit 1976 ihr Publikationsorgan, die HNO Informationen ( $\bullet$ Abb. 1), die auch im Besitz des Autors sind. Ein vertiefendes Quellenstudium des Autors in der Geschäftsstelle war wegen der COVID19-Pandemie nicht möglich. Jedoch erfolgte durch die Mitarbeiterinnen aus der Geschäftsstelle eine wesentliche und

Wolfgang Pirsig

Ulm, Deutschland

\title{
Zur internationalen Vernetzung der Deutschen Gesellschaft für Hals-Nasen-Ohren-Heilkunde, Kopf- und Hals-Chirurgie
}

kompetente Unterstützung der Recherche. Weil die Aktenunterlagen über die Gesellschaft seit der Gründung 1921 bis zum Jahr 1975 nicht durchgängig vorhanden waren, kamen letztlich nur partielle Ergebnisse über die hundertjährige Existenz der Gesellschaft zutage.

\section{\) Die Aktenunterlagen seit der Gründung 1921 bis zum Jahr 1975 waren nicht durchgängig vorhanden}

Ausgewertet wurden Anzeigen über angekündigte jährliche Treffen zwischen ausländischen und deutschen HNO-Gesellschaften. Dabei zählte der Kontakt zu einer ausländischen HNO-Gesellschaft nur einmal pro Jahr, auch wenn weitere Treffen stattfanden. Über die Realisierung dieser angekündigten Treffen gibt es keine durchgängigen Belege. Um auch etwas über andere internationale Kontakte zu erfahren, wurden Informationen über 210 Ehrenmitglieder bis 2020 und 140 lebende und 72 verstorbene korrespondierende Mitglieder aus 212 Ländern bis 2020 gesichtet. Bei den internationalen HNO-Dachorganisationen und Subspezialitäten wurde ebenfalls nach ihren Gründungsjahren und/oder Tagungsstätten recherchiert. Abschließend wurde kurz auf einige internationale Kooperationen der ehemaligen Deutschen Demokratischen Republik (DDR) von 1950 bis 1989 eingegangen. Informationen über diese Kooperationen stammen aus der Dissertation von Renate Lerch:
Zur Geschichte der Gesellschaft für OtoRhino-Laryngologie und zervikale Chirurgie der DDR [1].

\section{Vernetzungen seit 1921}

Die Gesellschaft Deutscher Hals-, Nasen- und Ohrenärzte war schon vor der Zusammenlegung der Deutschen Otologischen Gesellschaft mit dem Verein Deutscher Laryngologen 1921 international gut vernetzt. Das gilt besonders für einige Nachbarn Deutschlands wie Österreich, Tschechoslowakei, Schweiz, Italien, Niederlande, Luxemburg, Belgien, Frankreich und die europäischen Nordstaaten. Internationaler Austausch neuer Erkenntnisse erfolgte besonders in dem 1926 in Groningen (Niederlande) gegründeten Collegium Oto-Rhino-Laryngologicum Amicitiae Sacrum (CORLAS) [2]. Zu dessen Stiftungsmitgliedern zählten auch die Deutschen Otto Voss und Karl Wittmaack. In dieser Gesellschaft gehörte neben Englisch und Französisch auch Deutsch zu den offiziellen Sprachen. Das Collegium traf sich mit Ausnahme der Kriegsjahre jährlich und tagte 1930 unter Otto Voss in Frankfurt, 1964 unter Horst Wullstein in Würzburg und 1986 in München unter Hans Heinz Naumann.

Internationales wissenschaftliches Zusammenarbeiten von HNO-Ärzten in den 1920er-Jahren lässt sich auch in den Publikationen der HNO-Weltliteratur erkennen. Bei Wittmaack in Hamburg forschten beispielsweise in den 1920erund 1930er-Jahren Ärzte aus Europa, Japan und den USA. Das hörte ab 1933 


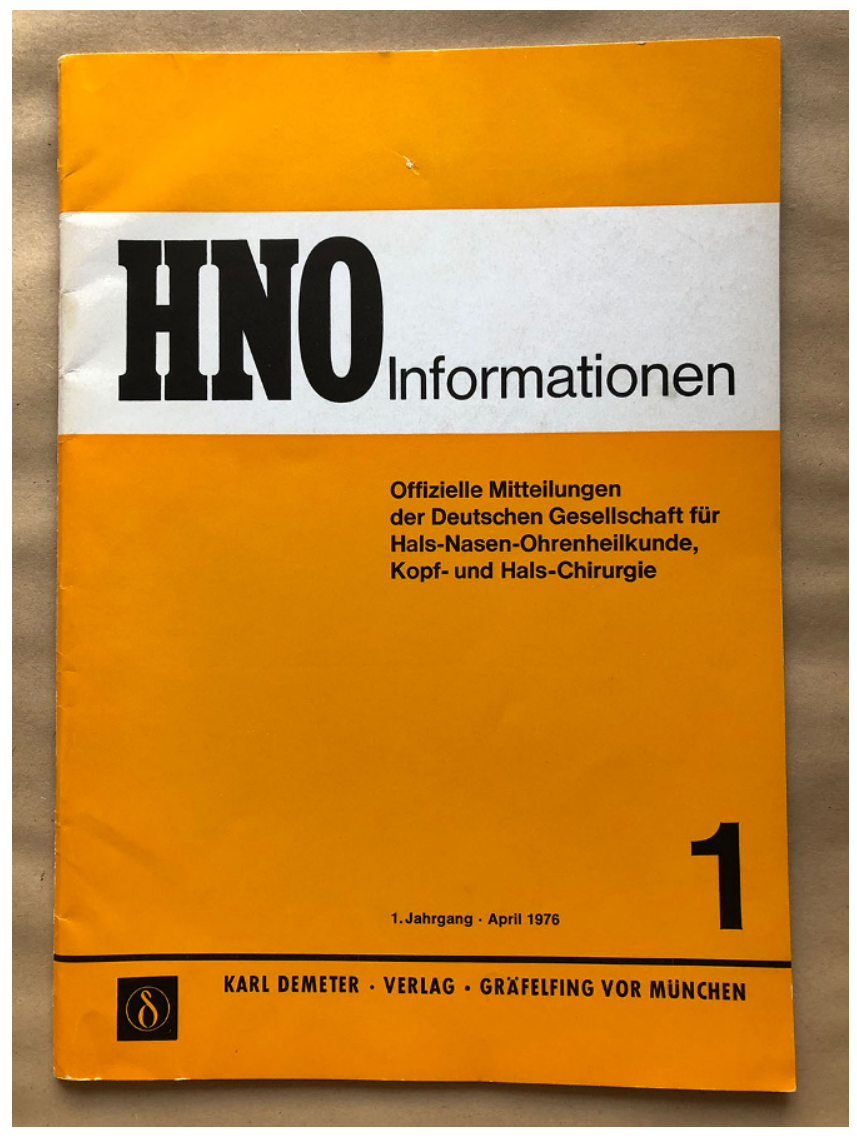

Abb. $1<$ Deckblatt der HNO Informationen 1 - April 1976. (Mit freundl. Genehmigung $\odot$ Georg Thieme Verlag KG, alle Rechte vorbehalten) in der Zeit der Nationalsozialisten auf. Im Jahr 1933 wurden alle jüdischen Professoren aus deutschen Universitäten verbannt und der Erwerb von internationalem Schrifttum partiell eingestellt. Daran änderte auch der Internationale Otorhinolaryngologie(ORL)-Kongress 1936 in Berlin nichts. Die letzte offizielle Jahresversammlung der Gesellschaft Deutscher Hals-Nasen-Ohrenärzte fand 1939 in Wien und die Kriegssondertagung 1943 in Breslau statt. Im Jahr 1945 ruhte der Postverkehr, und die Gesellschaft wurde - wie alle Vereinigungen in Deutschland - durch die Alliierte Militärregierung aufgelöst. Alle ihre Geschäftsunterlagen seit 1921 wurden im Zweiten Weltkrieg - außer dem Bericht über die Wiener Jahresversammlung zerstört.

Am 17./18.12.1946 wurde in der sowjetischen Besatzungszone Deutschlands in Berlin die deutsche Oto-RhinoLaryngologen-Tagung unter der Leitung von Carl Otto v. Eicken abgehalten, an der etwa 60 Fachkollegen teilnahmen. „Diese Fachtagung war nach 7jähriger
Pause die erste ihrer Disziplin“ [1, S. 23]. Gäste waren 3 HNO-Professoren aus der Sowjetunion. Im Jahr 1954 trafen sich in London wieder Mitglieder des Collegiums mit deutscher Beteiligung. Seine Mitglieder und Versammlungen bis 2001 haben zuletzt Huizing und van den Broek zusammengestellt [2].

\section{》) Im Jahr 1945 wurde die Gesellschaft durch die Alliierte Militärregierung aufgelöst}

Im Jahr 1949 fand die Neugründung der Gesellschaft Deutscher Hals-Nasen-Ohrenärzte in Karlsruhe statt, die wieder durch den Alterspräsidenten Carl Otto v. Eicken eröffnet wurde. Auch Kollegen aus der sowjetischen Besatzungszone konnten an der Tagung teilnehmen. Deren Teilnahme wurde mit der Gründung der DDR 1949 und besonders nach dem Mauerbau 1961 immer schwieriger. In Karlsruhe trafen sich auch Vertreter ausländischer HNO-Gesellschaften aus den 7 Ländern Österreich, Schweiz, Schwe- den, Griechenland, Luxemburg, Dänemark und Großbritannien ([3]: 2/19772). Auch für die folgenden 25 Jahre bis 1976 gibt es keine kontinuierlichen Unterlagen über die Entwicklung der neu gegründeten Gesellschaft Deutscher HalsNasen-Ohrenärzte. Es sind nur punktuelle Informationen über internationale Kontakte im Rahmen ihrer Jahresversammlungen von 1950 in Bad Kissingen bis 1975 in Wiesbaden verfügbar. Deren Erörterung würde den Rahmen dieser kurzen Übersicht jedoch sprengen. Anders sieht das für die Gesellschaft für OtoRhino-Laryngologie und zervikale Chirurgie der DDR aus. Darüber wird im Abschnitt „Internationale Vernetzung der Otorhinolaryngologie in der DDR" berichtet. Der breite HNO-Strom von 1921 war ab 1933 bis zum Kriegsende 1945 in eine nationale Röhre gepresst worden, in der internationale Kontakte zur Ausnahme wurden. Danach flossen $2 \mathrm{HNO}$-Ströme, Bundesrepublik Deutschland (BRD) und DDR, durch Deutschland, die ab 1961 noch durch eine zerteilende Mauer isoliert wurden. Erst 1990 wurden diese beiden Ströme wieder vereinigt.

\section{Vernetzungen seit 1976}

Erst seit 1976 gibt es mit dem Erscheinen der HNO Informationen für die BRD wieder regelmäßige Daten über internationale HNO-Kontakte. So fand zum Beispiel 1977 in Montpellier (Frankreich) die Sitzung der Fachsektion „Otorhinolaryngologie“ der Europäischen Vereinigung der Fachärzte statt. Dieser gehörten Belgien, Dänemark, Bundesrepublik Deutschland, Frankreich, Großbritannien, Irland, Italien, Luxemburg und die Niederlande an ([3]: 3/1978-1). Im Folgenden werden internationale HNOKontakte, die „fruchtbaren Nebenflüsse“ für den großen Strom DGHNO-KHC, als typische Beispiele in 2-Jahres-Blocks aus 5 aufeinanderfolgenden Jahrzehnten aufgelistet, um etwas über Schwankungen der jährlichen Kontakte zu erfahren. Die außereuropäischen Länder sind dabei durch Kursivsetzung markiert.

1976: 15 Kontakte zu Frankreich CSSR (Tschechoslowakische Sozialistische Republik) - USA - Portugal Dänemark - Belgien - Schweiz - Öster- 
reich - Griechenland - Polen - DDR Italien - Israel - Mexiko und Japan.

1977: 14 Kontakte zu Kanada - Argentinien - Polen - Italien - Mexiko DDR - Österreich - Ungarn - Dänemark - Israel - USA - CSSR - Schweiz und Niederlande.

1984: 16 Verbindungen zu Indien - USA - Österreich - Niederlande Malaysia - Rumänien - Spanien - Dänemark - Frankreich - Belgien - UK (Vereinigtes Königreich) - Kanada Schweiz - CSSR - Polen und Bulgarien.

1985: 13 Kontakte zu Frankreich USA - Israel - Japan - Kanada - Schweden - Belgien - UK - Österreich - CSSR Korea - Ungarn und Venezuela.

1992: 14 Kontakte zu USA - UK Frankreich - Mexiko - Österreich - Australien - Italien - Israel - Griechenland - Ungarn - Polen - Belgien - Schweiz und UdSSR (Union der Sozialistischen Sowjetrepubliken).

1993: 16 Verbindungen zu Frankreich - Italien - USA - Österreich Dänemark - Schweden - Polen - Spanien - Schweiz - Türkei - CZ (Tschechien) -Togo - UK - Griechenland - Australien und Israel.

2004: 9 Kontakte zu Polen - Griechenland - Frankreich - Niederlande Schweiz - Österreich - Australien - USA und Neuseeland.

2005: 12 Verbindungen zu Italien Schweiz - Niederlande - Australien CZ - UK - Litauen - Österreich - Griechenland - USA - Türkei und Neuseeland.

2016: 17 Verbindungen zu Niederlande - UK - CZ - Schweden - Schweiz Italien - Belgien - Österreich - Portugal - Spanien - Türkei - Frankreich Griechenland - Kanada - Republik Südafrika - Neuseeland und Hongkong.

2017: 14 Kontakte zu Österreich - Vereinigte Arabische Emirate - Niederlande Italien - UK - Polen - Frankreich - USA Spanien - Schweiz - Südkorea - Hongkong - Myanmar und Portugal.

Für die Jahre 2018 und 2019 hatten die Mitarbeiterinnen der Bonner Geschäftsstelle die Zählungen übernommen und kamen auf 24 europäische und 24 außereuropäische Länder, mit denen die DGHNO-KHC Verbindungen hatte ([4]: 44/2019-3).

HNO 2021 · 69:416-421 https://doi.org/10.1007/s00106-021-01013-4

(c) Springer Medizin Verlag GmbH, ein Teil von Springer Nature 2021

\section{W. Pirsig}

\section{Zur internationalen Vernetzung der Deutschen Gesellschaft für Hals-Nasen-Ohren-Heilkunde, Kopf- und Hals-Chirurgie}

\section{Zusammenfassung}

Um eine partielle Übersicht über die Vernetzung der Deutschen Gesellschaft für Hals-Nasen-Ohren-Heilkunde, Kopf- und Hals-Chirurgie (DGHNO-KHC) in der Welt zu gewinnen, wurden die Häufigkeiten ihrer jährlich angekündigten Kontakte mit internationalen HNO-Institutionen zwischen 1976 und 2020 anhand ihrer gelisteten Treffen in den HNO Informationen zusammengestellt. Von den 534 internationalen Treffen fanden 388 Treffen innerhalb und 146 außerhalb Europas statt. Diese Kontakte nahmen bis heute auch bis in weit entfernte Länder deutlich zu. Ferner wurden Ehrenmitglieder und korrespondierende Mitglieder der DGHNO-KHC gezählt. Bis 2020 gab es 210 Ehrenmitglieder, davon 53 lebende aus europäischen und 19 aus außereuropäischen Ländern. Bei den verstorbenen Ehrenmitgliedern (1951-2019) waren 125 aus Europa und 13 aus außereuropäischen Ländern. Bis 2020 wurden ferner 140 lebende und 72 verstorbene korrespondierende Mitglieder aus 212 Ländern gezählt, davon 143 aus europäischen und 69 aus außereuropäischen Ländern. Auch auf mehrere fachbezogene Subspezialitäten und HNO-Dachorganisationen besonders in Europa wurde eingegangen. Den Abschluss bilden einige internationale Kooperationen der ehemaligen Deutschen Demokratischen Republik von 1950 bis 1989. Insgesamt ergibt sich aus allem eine dichte weltweite Vernetzung der DGHNO-KHC in den letzten 5 Jahrzehnten.

\section{Schlüsselwörter}

Soziale Netzwerkanalyse · Medizinische Gesellschaften · Berufsorganisationen . Gesundheitssystembezogene Ökonomie und Organisationen · Europa

\section{On the international networking of the German Society of Otorhinolaryngology, Head and Neck Surgery}

\section{Abstract}

In order to obtain a partial overview of the integration of the German Society of Otorhinolaryngology, Head and Neck Surgery (DGHNO-KHC) in worldwide networks, the frequencies of its annually announced contacts with international otorhinolaryngologic societies between 1976 and 2020 were collected based on the meetings listed in the journal HNO Informationen. Of the 534 international meetings, 388 took place within and 146 outside Europe. These contacts have increased significantly to date, even in very distant countries. Furthermore, honorary and corresponding members of the DGHNO-KHC were counted. Up until 2020 there were 210 honorary members, 53 living honorary members from European countries and 19 from non-European countries. Among the deceased honorary members
(1951-2019), 125 were from Europe and 13 from non-European countries. Up until 2020, 140 living and 72 deceased corresponding members from 212 countries were counted, 143 from European and 69 from nonEuropean countries. Several subject-related subspecialties and otorhinolaryngologic umbrella organizations, particularly Europe, are also addressed. The last section concerns international cooperations of the former German Democratic Republic from 1950 to 1989. Overall, a dense worldwide networking of the DGHNO-KHC has been present during the past five decades.

\section{Keywords}

Social network analysis - Medical societies . Professional organizations - Health care economics and organizations - Europe
Zusammengefasst lässt sich aus allen Veranstaltungslisten belegen, dass zwischen 1976 und 2019 die DGHNO-KHC 534 internationale Kontakte $\mathrm{zu}$ anderen Ländern hatte: Dabei lagen 388 Länder innerhalb und 146 außerhalb Europas. Interpretiert man diese Zahlen mit dem
Bild des großen Stroms, so wurde dieser von gut doppelt so vielen europäischen Zuflüssen wie von außereuropäischen Quellen vergrößert. Betrachtet man die Verteilungen in einzelnen Jahrzehnten, so liegen die Zahlen in den Jahren von 1976 bis 1993 und von 2009 bis 2019 
deutlich höher als in den anderen Jahrzehnten. Bemerkenswert ist, dass auch so viele weit entfernte Länder der Welt in diese Kontakte eingebunden waren.

\section{Ehrenmitglieder und korrespondierende Mitglieder der DGHNO-KHC, HNO- Dachorganisationen und Subspezialitäten}

In den Gruppen der Ehrenmitglieder und korrespondierenden Mitglieder der DGHNO-KHC spiegelt sich ebenfalls die Bedeutung internationaler Kontakte wider. Auch für diese gelisteten Kontakte sei darauf hingewiesen, dass sie unvollständig sind, da sie nur aus den Veröffentlichungen in den HNO Informationen stammen.

Bis 2020 gab es 210 Ehrenmitglieder: 53 lebende Mitglieder aus europäischen (davon 24 deutsche) und 19 aus außereuropäischen Ländern. Bei den verstorbenen Ehrenmitgliedern (1951-2018/2019) waren 125 aus Europa und 13 aus außereuropäischen Ländern.

Von den 140 korrespondierenden Mitgliedern gab es bis 2020: 91 aus europäischen und 49 aus außereuropäischen Ländern. Bei den verstorbenen korrespondierenden Mitgliedern waren es 52 aus europäischen und 20 aus außereuropäischen Ländern (Bonner Geschäftsstelle 09.01.2021). Ehrenmitglieder und korrespondierende Mitglieder kamen aus einem der folgenden Länder: Ägypten, Argentinien, Australien, Belarus, Belgien, Brasilien, Bulgarien, Chile, China, Dänemark, Deutschland, Finnland, Frankreich, Georgien, Griechenland, Großbritannien, Irland, Italien, Japan, Kanada, Korea, Lettland, Luxemburg, Niederlande, Norwegen, Österreich, Peru, Polen, Ruanda, Rumänien, Russland, Schweden, Schweiz, Slowakei, Spanien, Südafrika, Tschechische Republik, Türkei, Ukraine, Ungarn und USA.

Weitere Informationen über internationale Vernetzungen der DGHNOKHCfinden sich auch in Ankündigungen über Gründungen und regelmäßige Treffen von bilateralen und multilateralen HNO-Gesellschaften. Ihre Aufzählung in der Welt würde mehrere Seiten füllen. Hier werden beispielsweise einige für
Deutschland mit punktuell herausgegriffenen Jahresdaten aufgelistet:

- UEMS-ORL (Union Européenne des Médecins Spécialistes, Section ORL) von 1958 in Brüssel (Belgien)

- IFOS (International Federation of Otorhinolaryngological Societies) 1965 in Tokio (Japan), 2013 in Seoul (Südkorea)

- DONAU-SYMPOSIUM seit 1978 in Prag (Tschechien), 1984 in Graz (Österreich). Es fand statt: 1986 in Düsseldorf (am Rhein!) mit der BRD, DDR, Österreich, Tschechoslowakei, Ungarn, 1990 in Budapest (Ungarn), 2014 in Cluj-Napoca/Klausenburg (Rumänien) und das 14. Mal an der Donau 2018 in Krems (Österreich)

- ESPO (European Society of Pediatric Otorhinolaryngology) seit 1977 als EWGPO (European Working Group in Pediatric Otorhinolaryngology) und seit 1994 als ESPO, 2012 in Amsterdam (Niederlande)

- EUFOS (European Federation of Otorhinolaryngological Societies) von 1985 in Paris (Frankreich), Barcelona (Spanien) 2011. Auf dem Treffen 2000 in Berlin wurde u. a. mit 11 Postern die Basis für eine „History of otorhinolaryngology in different European countries between 1880 and 1920" gelegt [5]

- EAORL-HNS (European Academy of Otorhinolaryngology, Head and Neck Surgery) von 2005 in Marburg

- CEORL-HNS (Confederation of European Societies of Otorhinolaryngology, Head and Neck Surgery) 2011 in Barcelona

Spezielles Fachwissen wurde auch in vielen internationalen Gesellschaften ausgetauscht, von denen hier eine kleine Auswahl mit stets nur einigen Tagungsstätten vorgestellt wird:

- Bárány Society, gegründet 1960, 1978 in Uppsala (Schweden)

- ERS (European Rhinologic Society) seit 1964, in Erlangen 1975, 2002 in Ulm, 2011 in Tokio und 2016 in Stockholm (Schweden)

- ESBS (European Skull Base Surgery) von 1979, 2005 wieder in Fulda

- ELS (European Laryngological Society) gegründet 1995 in Brüssel,
13. (virtueller) Kongress 2021 in Berlin

- EAONO (European Academy of Otology and Neuro-Otology) 2006 in Köln

- ESSD (European Society for Swallowing Disorders) mit 10. (virtuellem) Kongress Oktober 2020

- EAFPS (European Academy of Facial Plastic Surgery) von 1977, 34. Konferenz in Brügge (Belgien) 2011

- UEP (Union of European Phoniatrics) von 1973, 26. Kongress 2011 in Lund (Schweden)

- Politzer Society 1. internationale Konferenz in Davos (Schweiz) 1978, 32. Treffen 2019 in Warschau (Polen)

Auch bilaterale HNO-Gesellschaften zwischen einer Nation und der BRD ermöglichten erfolgreiche Vernetzungen von Nationen wie die Deutsch-Französische HNO-Gesellschaft, die seit 1981 in Rennes (Frankreich) alle 2 Jahre, 2014 in Lyon (Frankreich) und zuletzt virtuell vernetzt in Tübingen 2020 tagte. Die Deutsch-Spanische Gesellschaft für HNO-KHC traf sich seit 1994. Im Jahr 2010 tagte sie mit der Peruanischen HNO-Gesellschaft in Iquitos (Peru) und 2016 in Düsseldorf. Das 1st Joint Meeting der DGHNO-KHC mit der American Academy of Otorhinolaryngology Head and Neck Surgery (AAO-HNS) fand 2006 in Mannheim statt. Seitdem wurde bei allen Jahresversammlungen ein englischsprachiger Teil für Besucher aus dem jeweiligen Gastland durchgeführt, wie beispielsweise 2012 für die Deutsch-Chinesische HNO-Gesellschaft (CGSO-HNS) in Mainz. Die DeutschTschechische HNO-Gesellschaft traf sich 2010 in Bad Salzungen, 2012 in Leipzig und 2016 in Hamm.

Eine herausragende Stellung bei den vernetzenden internationalen Aktivitäten haben die Mitglieder aus der Interessengemeinschaft humanitär im Ausland tätiger HNO-Ärzte inne, deren Aktivitäten sich über viele Länder auf mehreren Erdteilen erstrecken. 


\section{Internationale Vernetzung der Otorhinolaryngologie in der DDR}

Dieser Bericht endet mit einigen Informationen über die Otorhinolaryngologie in der DDR, mit der die BRD von 1950 bis 1989 in einer sehr speziellen Art vernetzt war. Frau Dr. Lerch hat über die Gesellschaft für Oto-Rhino-Laryngologie und zervikale Chirurgie der DDR sehr detailliert nationale und internationale Kongresse und Kooperationen aufgelistet. In diesen Listen finden sich auch einige Aufzeichnungen über Kongresse in der BRD aus den Jahren 1950 bis 1975 . Hier folgen einige Beispiele von internationalen Kooperationen der DDR von 1948 bis in die 1980er-Jahre:

- 1948 Internationale Tagung der Audiologen in Stockholm mit BRD und DDR

- 1952 CORLAS in Zürich, als Deutsche erstmals nach dem Krieg wieder eingeladen wurden

- 1965 Erstes internationales Symposium für Cochlea-Forschung in Halle (nachfolgend alle 3 Jahre mit internationaler Beteiligung)

- 1970 Gründung der Europäischen Aerosolgesellschaft mit Prof. Zippel/ Greifswald

- 1972 Österreichischer HNO-Kongress in Salzburg

- 1973 Internationaler Otorhinolaryngologischer Kongress in Venedig

- 1974 Bilaterales Symposium zwischen DDR und Polen (Audiologie, Cochlea)

- 1975 Schwedischer ORL-Kongress in Stockholm

- 1979 DDR und RWG-Staaten in Tbilissi/Tiflis (Georgien), (RWG: Rat für gegenseitige Wirtschaftshilfe osteuropäischer Staaten)

- 1980 Audiologie-Symposium von Ungarn und der DDR

- 1981 Internationaler Otorhinolaryngologischer Kongress in Budapest

- 1981 Italienischer HNO-Kongress in Triest

- 1981 Gemeinschaftstagungen von Leipzig, Olomouc/Olmütz (Tschechien) und Szeged (Ungarn) in Ungarn
Es gab klare Vorstellungen der DDRRegierung, mit welchen ausländischen HNO-Gesellschaften man zusammenarbeiten sollte: nämlich mit den Ländern der „Wirtschaftsvereinigung der osteuropäischen Staaten“. Eine Quelle dazu ist das Ergebnis einer Arbeitsberatung des Vorstands der Gesellschaft mit dem Generalsekretariat der medizinisch-wissenschaftlichen Gesellschaften der DDR und Vertretern des Ministeriums für Gesundheitswesen: „Am 15.11.1975 wurden für die Gestaltung der internationalen Beziehungen Schwerpunkte gesetzt: die Beziehungen zu Fachgesellschaften der UdSSR und der anderen sozialistischen Staaten seien vorrangig zu entwickeln. Weiterhin sollte den Kontakten zu Entwicklungsländern mehr Bedeutung als zu kapitalistischen Industriestaaten beigemessen werden.“ [1, S. 151].

\section{》) Es gab klare Vorstellungen der DDR-Regierung, mit wem man zusammenarbeiten sollte}

Die DDR war auch in folgenden internationalen Gesellschaften und Organisationen vertreten: Bárány-Gesellschaft, European Working Group in Pediatric Otorhinolaryngology, International Association of Logopaedics and Phoniatrics, International Committee of Speech and Hearing, Internationale Gesellschaft für Innenohrbiologie, Internationale Gesellschaft für Audiologie, Internationale Gesellschaft für Neuro-Otologie und Äquilibriometrie und International Skull Base Study Group [1, S. 149].

Zusammengefasst ergibt sich aus dieser kurzen Übersicht eine dichte weltweite Vernetzung der DGHNO-KHC. Der lange Strom ist durch seine zahlreichen Nebenflüsse aus der ganzen Welt seit 1921 immer breiter geworden. Neues Land für die Hals-Nasen-Ohren-Heilkunde, Kopf- und Halschirurgie wurde dadurch in seiner Mündung geschaffen, auf das die in diesen Bereichen Tätigen bauen können.

\section{Fazit für die Praxis}

- Die Deutsche Gesellschaft für HalsNasen-Ohren-Heilkunde, Kopf- und Hals-Chirurgie (DGHNO-KHC) verfügte über eine dichte weltweite Vernetzung in den letzten 50 Jahren.

- Auf Grundlage der Zeitschrift HNO Informationen 1976 bis 2020 wurden die Ankündigungen der DGHNOKHC für Kontakte mit internationalen HNO-Institutionen sowie die Herkunft der Ehren- und der korrespondierenden Mitglieder ausgewertet.

- So fanden 534 Treffen auf internationaler Ebene statt (388 innerhalb und 146 außerhalb Europas).

- Bei den bis 2019 verstorbenen Ehrenmitgliedern waren 125 aus Europa und 13 aus außereuropäischen Ländern, bei den lebenden 53 aus Europa und 19 aus außereuropäischen Ländern.

- Es gab 140 lebende und 72 verstorbene korrespondierende Mitglieder aus 212 Ländern.

- Wie die Auswertung der Dissertation von Renate Lerch an der Charité zeigt, lag der Fokus der Otorhinolaryngologie in der DDR auf der Zusammenarbeit mit Ländern der „Wirtschaftsvereinigung der osteuropäischen Staaten".

\section{Korrespondenzadresse}

Prof. Dr. med. Wolfgang Pirsig

Mozartstraße 22/1, 89075 Ulm, Deutschland wolfgang.pirsig@uni-ulm.de

Danksagung. Frau Ulrike Fischer und Frau Monika Thorleuchter von der Bonner Geschäftsstelle der DGHNO-KHC danke ich ganz herzlich für die stetige Unterstützung bei der kniffligen Ländersuche für diesen Bericht. Mein besonderer Dank gilt Frau Dr. Renate Lerch aus Berlin. Ohne ihre herausragende Dissertation aus der Charité hätte ich einige spezielle historische Zusammenhänge nicht darstellen können.

\section{Einhaltung ethischer Richtlinien}

Interessenkonflikt. W. Pirsig gibt an, dass kein Interessenkonflikt besteht.

Für diesen Beitrag wurden von dem Autor keine Studien an Menschen oder Tieren durchgeführt. Für die aufgeführten Studien gelten die jeweils dort angegebenen ethischen Richtlinien. 


\section{Literatur}

1. Lerch R (1990) Zur Geschichte der Gesellschaft für Oto-Rhino-Laryngologie und zervikale Chirurgie der DDR. Dissertation Charité Berlin

2. Huizing EH, van den BroekP(2002) Vivat Collegium Oto-Rhino-Laryngologicum Amicitiae Sacrum 1926-2001. Coers en Roest, Arnhem

3. HNO Informationen (2006) 1. Jahrgang 1976 bis 31. Jahrgang 2006. Karl Demeter, Gräfelfing vor München

4. HNO Informationen (2020) 32. Jahrgang 2007 bis 45. Jahrgang 2020. Deutscher Ärzteverlag, Köln

5. Mudry A, Pirsig W, Weir N (2005) History of otorhinolaryngology (ORL) in different European countries between 1880 and 1920. J Laryngol Otol 119(Suppl No 30):1-2. https://doi.org/10.1258/ 0022215054527401

\section{W. Pirsig, R.J. Ruben (Eds.)}

\section{The Case of Frederick the Noble}

Felix Semon's letters to N.H. von Bismarck

Amsterdam: Kugler 2020, 68 S., 16 Abb., (ISBN: 978-90-6299-285-0), Softcover/eBook 20,00 EUR

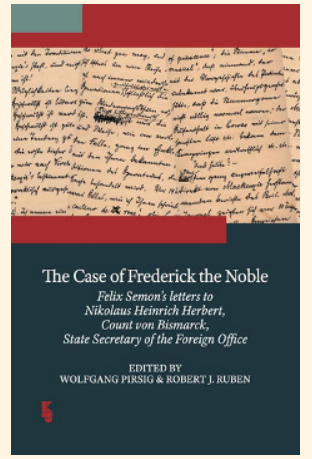

In Zeiten von Krisen hilft die Rückbesinnung auf Erfahrungen aus der Geschichte. Gewonnene Zeit durch eingeschränkten Bewegungsradius macht es möglich, ein gutes Buch in die Hand zu nehmen. Wer historisch interessiert ist und vor englischsprachiger Lektüre nicht zurückschreckt, dem sei dieses kleine Büchlein über die Irrungen unserer fachlichen Vorfahren bei der Behandlung des preußischen Thronfolgers und späteren Kaisers Friedrich III. empfohlen.

Anhand des Briefwechsels von Felix Semon, dem wegen jüdischer Herkunft aus Berlin ins Londoner Exil gegangenen deutschen Laryngologen und dem Sohn Otto von Bismarcks eröffnet das von Wolfgang Pirsig und Robert J. Rubin liebevoll zusammengestellte Zeitdokument einen wunderbaren Einblick in die kollegiale Wahrnehmung der Behandlung des berühmten Patienten durch den anerkanntesten Laryngologen dieser Zeit, Sir Morell Mackenzie.

Interessant war die Lektüre für mich aus mehrerlei Gründen. Zum einen wollte ich mehr über die Person von Felix Semon erfahren, der 1881 das Semon-Rosenbachsch'e Gesetz zur Stimmlippenstellung bei Rekurrensparesen begründete. In der Sprache seiner Briefe spiegelt sich die blumige Ausdrucksweise seiner Fachpublikationen wider. Semon war ein brillanter Beobachter und Beschreiber von Krankheitsverläufen. Auf diese Weise arbeitete er Gesetzmäßigkeiten und Abweichungen von der Norm heraus, wie dies in dieser Tiefe heute nicht mehr zu beobachten ist.
Zum anderen interessierte mich der Entwicklungsstand der Laryngologie in dieser frühen Phase unseres Fachgebietes. Wenn wir uns heute über eine zu deskriptive Histologie ohne klinische Konsequenz ärgern, sollten wir bedenken, dass auch ein Virchow sich irren konnte oder die dem Pathologen vorgelegte Probe nur einen Teil der Wahrheit repräsentierte. Operative Eingriffe, die für uns heute selbstverständlich sind, galten Ende des 19. Jahrhunderts noch als experimentell und zu über 50\% tödlich. Schon 10 Jahre nach dem Tod Friedrich III. konnten Kehlkopfteilresektionen von außen und Laryngektomien mit vertretbaren Überlebenschancen ausgeführt werden. Sicherlich hat die Erkrankung des berühmten Patienten die weitere Entwicklung der Larynx-Chirurgie herausgefordert und beflügelt.

Schließlich fand ich interessante Parallelen zur Gegenwart. Schon damals glaubten Kollegen, die nicht selbst an der Behandlung beteiligt waren zu wissen, wie die Dinge ausgehen würden. Die Gefangenheit von im Rampenlicht stehenden Experten in einmal getroffene Entscheidungen, ihre begrenzte Kritikfähigkeit und ihr Fleiß der Rechtfertigung in Publikationen und Fachvorträgen zeigen die Schwächen der „Eminenz" basierten Medizin auf. Wenn heute Assistent ${ }^{*}$ Innen während der Chefvisite getroffene Entscheidungen am Smartphone einem „FaktenCheck" unterziehen, dann sollte man dies nicht persönlich nehmen. Eine für Kritik offene Atmosphäre und die Einbeziehung aller geäußerten Meinungen schafft Transparenz und Akzeptanz, insbesondere wenn die Erkrankung unerwartet einen ungünstigen Verlauf nimmt.

In diesem Sinne wünsche ich Ihnen viel Freude und Erkenntnis bei der Lektüre. Andreas Müller 